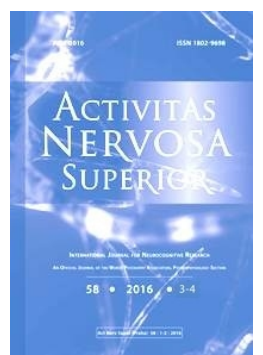

ANS: Journal for Neurocognitive Research Homepage:

WWW.activitas.org

\title{
GAMMA COHERENCES IN THE Default MODE Resting STATE as a MeAsure OF CONSCIOUSNESS LEVEL
}

\author{
Peter Jombik,,$^{*}$ Michal Drobny, ${ }^{2}$ Beata Saniova, ${ }^{2}$ Martin Fischer, ${ }^{2}$ Petra Kaderjakova ${ }^{1}$ \\ ${ }_{1}$ Department of Neurology, Hospital Zvolen, Slovak Republic \\ ${ }^{2}$ Department of Anaesthesiology and Intensive Medicine, Comenius University in Bratislava, \\ Jessenius Faculty of Medicine and University Hospital in Martin, Slovak Republic
}

\begin{abstract}
Recent findings indicate that the default mode resting state network (DMRSN) is closely associated with conscious awareness. Thus changes in consciousness level could be reflected in turn in the DMRSN functional state. One of the most important requirements for proper network function is connectivity within the network structural constituents. One of the measures of functional connectivity is analysis of the EEG coherences. In this study we have examined changes in the mean coherences in the gamma spectral band $30-45 \mathrm{~Hz}$ in the DMRSN during general anaesthesia (GA) in the open thorax surgery were investigated in twenty patients. They were compared with physiologic EEG findings in twenty alert subjects and the mathematical model of brain death. Results of this study indicate that the decrease in coherences in the physiologic EEG and the patients with one lung ventilation (OLV) groups was roughly proportional to the increasing distance between the electrodes. This created some kind of a structure in the DMRSN. In the OLV group the main finding was a decrease in the coherences in the gamma band. Ten of the anaesthetized subjects showed a clear burst suppression pattern. During this condition the coherences in the pairs connecting the posterior parts of the network decreased. Moreover, the differences between the connections of the anterior to posterior parts of the DMRSN and connections between the posterior parts of the DMRSN were almost lost. However, they showed still highly significant differences in all items when compared with the mathematical model of brain death. In summary the results suggest that the functional connectivity in the DMRSN assessed by mean coherences could be a reliable method for assessing the depth of GA and may be useful for understanding disorders of consciousness in general.
\end{abstract}

Key words: Functional connectivity; Default mode resting state network; Consciousness loss; Brain death model; OLV group; Flat group

\section{INTRODUCTION}

The default mode resting state network (DMRSN) constitutes a set of brain areas which are active in conditions when the subject is at rest, typically with eyes closed. In this condition the mental contents are delivered from internal sources, i.e. the subject's memory. They are

${ }^{*}$ Peter Jombik, email: peter.jombik@hotmail.sk

Received October 4, 2016; accepted November 10, 2016; Act Nerv Super 58(3-4), 71-77; ISSN-1802-9698 
subjected to the imaginary process. Attitudes are formed and future plans are created. The main areas that constitute this network are the following: the ventral medial prefrontal cortex, dorsal medial prefrontal cortex, posterior cingulate/retrosplenial cortex and the inferior parietal lobule (Buckner et al., 2008). The co-working between these spatially separated regions of the brain can be studied using methods which analyze the indices of connectivity in the EEG signals of spatially separated regions (Lehembre et al., 2012; Greenblatt et al., 2012; Sakkalis, 2011).

One such method is cross spectrum analysis, and one of its products, the squared coherence, can reflect functional connectivity. The squared coherence is the squared correlation coefficient between the cyclical components in two series at the respective frequency. It is the product of the cross-amplitude values after squaring them and dividing by the product of the spectrum density estimates for each series (Bowyer, 2016). It is well known that the squared coherence value decreases with increasing distances between the electrodes. The coherences can also show some variability over time, i.e. by changing the analysis epoch duration (Bullock et al., 1995).

The function of the DMRSN is altered in several pathological states (Buckner et al., 2008; Guldenmund et al., 2012). However, disorders of the consciousness would also interfere with the proper functioning of the DMRSN (Guldenmund et al., 2012). It has been shown that GA is associated with decrease in the coherences in several frequency bands (John et al., 2001). The medial prefrontal cortex and posterior cingulate/precuneus region have been found to be two of the brain areas most sensitive to anaesthetics (Vogt \& Laureys, 2005; Boveroux et al., 2010; Cascella, 2016). The observed changes in DMN connectivity during loss of consciousness and alteration of external awareness mean that DMN integrity may represent the level of consciousness. According to our hypothesis different levels of consciousness loss would be reflected in different levels of the DMRSN functional derangements, which in turn could be assessed by means of coherence analysis. GA can serve as a controlled model of consciousness loss. For this reason we studied subjects with normal EEG and patients in GA during the open thorax surgery.

\section{METHODS}

\subsection{Subjects}

The control group of waking subjects with physiologic EEG. EEG read-outs of 20 adults, roughly age-matched subjects (10 women and 10 men; mean age 51.63; SD 16.22; range 18-77 years) were analyzed. The data were retrospectively selected from our EEG database (Hospital Zvolen, Department of Neurology). The data constituted 19 channel EEG recordings in accordance with the international 10-20 system, referenced to the FAz electrode, sampled at $128 \mathrm{~Hz}$ and band-pass filtered at $0.5-45 \mathrm{~Hz}$. The criteria for inclusion were the following: normal EEG features on visual inspection by two independent examiners and normal indices of quantitative background analysis of the EEG sample (i.e. an epoch with duration of $8 \mathrm{sec}$ ), which represented the typical features of the subject's waking artefact-free EEG record. Only records of subjects who were not taking psychotropic medication were included to the study.

\subsection{EEG of patients during general anaesthesia}

EEG read-outs of 20 adult subjects in GA during the open thorax surgery - lung resection (10 women; 10 men; mean age 60; GA - Isofluran; Desfluran; Department of Anaesthesiology and Intensive Medicine, University Hospital in Martin). These data constituted 19 channel EEG recordings in accordance with the international 10-20 system, referenced to the $\mathrm{Cz}$ electrode, sampled at $500 \mathrm{~Hz}$ and band-pass filtered at $0.5-45 \mathrm{~Hz}$. In each of the 20 subjects one $(8 \mathrm{sec})$ EEG epoch was selected to further analysis. All these selected epochs were recorded during 
the key phase of the surgery, when a lung resection was done. This step of the thorax surgery was always associated with OLV. We assumed that in this step of the thorax surgery the depth of the anaesthesia was most appropriate and roughly comparable across the subjects. Moreover, in most of the anaesthetized subjects a clear burst-suppression pattern was present which was induced by intravenous application of propofol (intravenous anaesthetic agent) at the beginning of GA.

\subsection{Data analysis}

In 10 subjects the suppression length of the flat EEG (Flat) was enough long (i.e. not interrupted by episodes of the burst) to allow analysis as an $8 \mathrm{sec}$ long epoch. Thus, two different patterns of the EEG were subjected to analysis in the anaesthetized group: the OLV (20) and the Flat epochs (10).

In order to make the EEG data of both groups comparable, the data of control waking subjects and patients with dementia were re-referenced to $\mathrm{Cz}$ (Thatcher, 2012; Vattal et al., 2005), and the data of the patients recorded during anaesthesia were down-sampled to $128 \mathrm{~Hz}$.

Epochs with duration of $8 \mathrm{sec}$ after DC (Direct Current) shift and trend removal were subjected to Hann weighted analysis using FFT (Fast Fourier Transform). The FFT results of the whole scalp 19 electrode array were then used to obtain some derivatives of the quantitative EEG power-spectral analysis. These derivatives included the following indexes: diffused slowing in the band-pass $0.5-45 \mathrm{~Hz}$ and in the band-pass $2-25 \mathrm{~Hz}$, and the anteroposterior gradient of the alpha rhythm (Gotman et al., 1973; Lodder \& van Putten, 2013). Moreover, six electrode position pairs which represent the DMRSN assembly (Fz-Pz; Fz-P3; Fz-P4; Pz-P3; Pz-P4 and P3-P4) were subjected to Hamming weighted FFT and cross spectrum analysis with $\Delta \phi$ at 0.5 and $0.125 \mathrm{sec}$ for the $2 \mathrm{sec}$ and the $8 \mathrm{sec}$ epochs respectively. The initial segments of the EEG epochs with $2 \mathrm{sec}$ duration and the whole epochs with duration of $8 \mathrm{sec}$ were processed separately using the cross spectrum analysis method. Mean squared coherences were calculated in the gamma spectral band 30-45 Hz. The between-group differences in the quantitative EEG indexes of the background were assessed with KruskalWallis ANOVA. Differences between coherences of 2 sec epochs and the 8 sec epoch were investigated with the Wilcoxon matched pairs test. The between-group differences in the coherences in the $2 \mathrm{sec}$ epochs and the coherences in the $8 \mathrm{sec}$ epochs were estimated with the Mann-Whitney $U$ test. The statistical significance level was set to $p<0.05$. In general there were no significant differences between the coherences in the $2 \mathrm{sec}$ and the $8 \mathrm{sec}$ epochs (exceptions were in the OLV delta range of the Fz-P3; Pz-P4 and the P3-P4 pairs; in the Flat delta range of the Fz-P3; Pz-P4 pairs; the Flat alpha range Fz-P4 and the Flat theta range P3P4). Moreover, the $2 \mathrm{sec}$ epochs showed much higher variance than the $8 \mathrm{sec}$ epochs. Thus for conciseness, only the most reliable indices, the endpoint coherences in the 8 sec epoch are shown in the results.

\section{RESULTS}

\subsection{Quantitative EEG features}

All three global indexes, i.e. diffused slowing in the range $0.5-45 \mathrm{~Hz}$; diffused slowing in the range $2.0-25 \mathrm{~Hz}$; and the quantitative antero-posterior gradient of the alpha rhythm, were abnormal in the patient groups and significantly different from the control group. The Flat group showed significantly more diffused slowing in the $2-25 \mathrm{~Hz}$ range compared to the OLV group. Otherwise the differences between the OLV and the Flat groups were not significant. 


\subsection{General findings in linear regressions}

The coherences did not show any consistent change over time, i.e. between the short 2 sec and the long $8 \mathrm{sec}$ epochs. The coherences showed a decrease with increasing distance. This decrease in the coherences in the control and the OLV groups was roughly proportional to the increasing distance between the electrodes. The Pz-P3 and Pz-P4 pairs showed the highest coherences, in contrast to the Fz-P3 and Fz-P4 pairs with the lowest figures. Thus in the graphical illustration these figures created a pattern, i.e. some kind of "structure" in the DMRSN. This feature was lost in the Flat group. The separation of the different pairs tended to become almost negligible, and the "structure" collapsed, as will be described in more detail later (Figure 1).
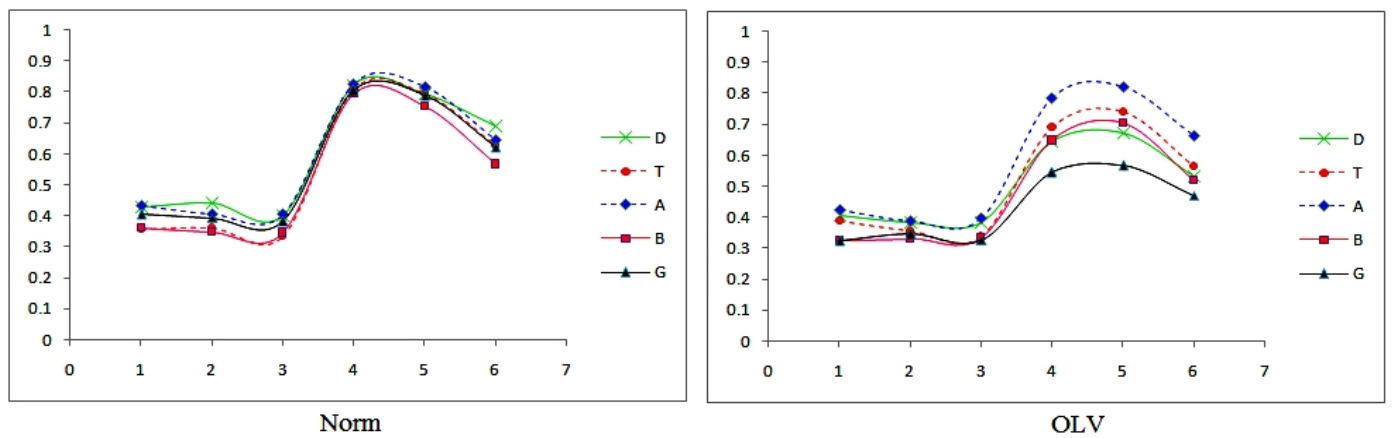

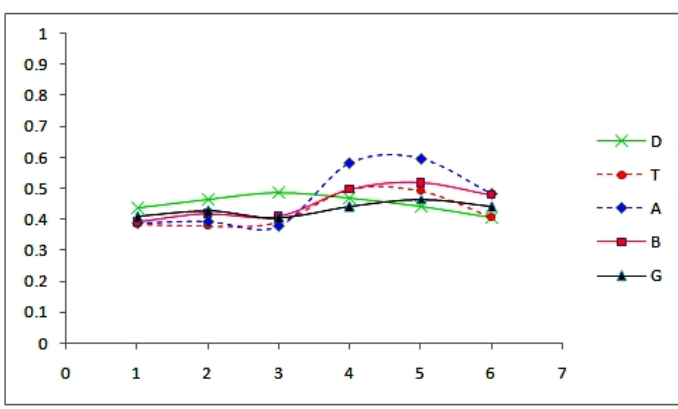

Flat

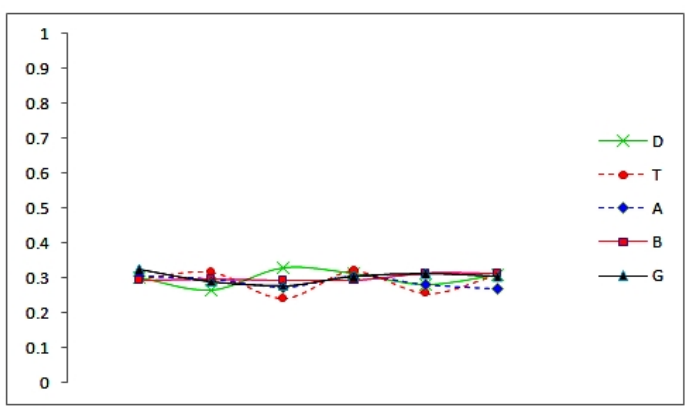

Death

Figure 1. Coherences in the DMRSN in the particular groups of patients and the brain death model; the marks on the traces refer to Fz-Pz; fz-P3; Fz-P4; Pz-P3; Pz-P4 and P3-P4 pairs; note that the decrease of the coherences is most pronounced in the posterior areas of the network, by increasing depth of consciousness loss.

\subsection{Differences between the groups.}

OLV vs. control group. The OLV group showed significantly lower coherences in the gamma band in the Pz-P3 and Pz-P4 pairs (Figure 1).

Flat vs. OLV group.

Differences with significantly lower coherences in the Flat group were almost consistently present in pairs with the shortest distances, connecting the posterior parts of the brain (Pz-P3; $\mathrm{Pz}-\mathrm{P} 4$ and P3-P4 pairs). Exceptions were the Fz-Pz and the P3-P4 pairs. In the pairs with longer distances connecting the anterior to the posterior parts of the brain the differences were less consistent, because in some of these pairs the coherences in the Flat group were even significantly higher (Figure 1). 
The differences between the short and long distance pairs in the Flat group. The differences between the short and long distance pairs were almost lost in the Flat group. All pairs regardless of the distance and the connectional topography, showed very similar figures (Figure 1).

Comparison of the Flat group with the mathematical model of brain death. The OLV and Flat groups compared to the control and dementia group showed some features of progressive increase in entropy. The coherences of the Flat group were therefore compared with those in the mathematical model of brain death. Brain death in this model was approximated by pseudo-random numbers produced by the random number generator in Excel. The plots of the Flat group and the brain death model were very similar. However, the statistical analysis showed highly significant differences in all pairs with lower coherences in the brain death model (Figure 1).

\section{DISCUSSION}

We could not reproduce the coherence decrease over time (Bullock, 1995). The reason for this might be methodological. Firstly, the variances of the signal in the surface recordings were much higher than in the recordings close to the source of the signal by depth electrodes. Secondly, the analysis time of $8 \mathrm{sec}$ was too short compared to $60 \mathrm{sec}$ in Bullock's study. The trends in the coherence decrease were roughly proportional to the distance between the electrodes. Anaesthetized patients who were unconscious showed significant decrease in coherences in the gamma range in the pairs connecting the posterior areas of the brain. Thus the coherence in the gamma band could be considered as one of the most sensitive indices of consciousness loss (John et al., 2001; Cavinato et al., 2015).

Moreover, this decrease was greatly pronounced in the deepest stage of the anaesthesia, i.e. in the flat epochs of the burst-suppression pattern. Here the differences between connections of the anterior to posterior areas and the connections between posterior areas of the DMRSN were almost lost. The graphical structure of the DMRSN collapsed, and it took on an appearance which was similar to the brain death model, but statistically it was still highly significantly different from that model. In a PET study by Alkire et al.,(1998) during propofol anaesthesia the mean cerebral metabolic rate was reduced by $38 \%$ at loss of consciousness, uniformly throughout the brain but more in the cortex than sub-cortex. In sedation, very similar but lesser global reduction was found. They concluded that loss of consciousness was not caused by changes within a specific circuit but rather by uniform reduction below a critical level in the distributed neural system (Alkire et al., 1998). Similar global quantitative results were found with benzodiazepine - midazolam (Veselis et al., 1997).

However, this study and also several other studies in anaesthesia or in the vegetative state (Fiset et al., 1999; Alkire et al., 2000; Laureys et al., 2000) showed that significantly greater reduction was seen in multiple regions of the prefrontal cortex, the superior frontal gyrus, the anterior cingulate gyrus, the parietal association areas, the insula and the thalamus. The majority of these areas constitute the DMRSN or have a close relationship to it. General anaesthesia is associated with hyper-coupling among regions of the prefrontal and frontal regions within each hemisphere, but uncoupling of the anterior from posterior regions within each hemisphere as well as of homologous regions between the hemispheres (John et al., 2001). This is in agreement with our results, which show a gradual increase in the degree of uncoupling, in the gamma band by increasing the depth of the anaesthesia (John \& Prichep, 2001). 


\section{CONCLUSION}

Analysis of the mean squared coherences in the DMRSN EEG could offer a reliable and robust method for quantitative assessment of depth of unconsciousness. Functional connectivity in the DMRSN assessed by mean coherences could be a reliable method for assessing the depth of general anaesthesia and maybe also disorders of consciousness in general.

\section{ACKNOWLEDGEMENTS}

The study was supported by the project Consciousness and its changes during general anaesthesia in thoracic surgery. APVV 0223/12. Basic research. Slovak Research and Development Agency.

\section{REFERENCES}

Alkire, M.T., Haier, R.J., \& Fallon, J.H. (1998). Toward the neurobiology of consciousness: Using brain imaging and anesthesia to investigate the anatomy of consciousness. In S. R. Hameroff, A. Kaszriak, \& A. Scott (Eds.), Toward a science of consciousness II - The second Tucson discussion and debates, pp. 255268. Cambridge, MA: MIT Press.

Alkire, M. T., Haier, R.J, \& Fallon, J. H. (2000). Toward a unified theory of narcosis: Brain imaging evidence for a thalamocortical switch of the neurophysiologic basis of anestheticinduced unconsciousness. Consciousness and Cognition, 9, 370-386.

Boveroux, P., Vanhaudenhuyse, A., Bruno, M.A., Noirhomme, Q., Lauwick, S., Luxen, A., Degueldre, C., Plenevaux, A., Schnakers, C., Phillips, C., Brichant, J.F., Bonhomme, V., Maquet, P., Greicius, M.D, Laureys, S., \& Boly, M. (2010). Breakdown of within- and between-network resting state functional magnetic resonance imaging connectivity during propofol-induced loss of consciousness. Anesthesiology, 113, 1038-1053.

Bowyer, S.M. (2016). Coherence a measure of the brain networks: past and present. Neuropsychiatric Electrophysiology, 2, 1-12.

Buckner, R.L, Andrews-Hanna, J.R, \& Schacter, D.L. (2008). The Brain's Default Network Anatomy, Function, and Relevance to Disease. Annals of the New York Academy of Sciences, 1124, 1-38.

Bullock, T.H., McClune, M.C., Achimowicz, J.Z., Iragui-Madoz, V.J., Duckrow, R.B, \& Spencer, S.S. (1995). EEG coherence has structure in the millimeter domain: subdural and hippocampal recordings from epileptic patients. Electroencephalography and clinical Neurophysiology, 95, 161-177.

Cavinato, M., Genna, C., Manganotti, P., Formaggio, E., Storti, S.F, Campostrini, S., Arcaro, C., Casanova, E., Petrone, V., Piperno, R. \& Piccione, F. (2015). Coherence and Consciousness: Study of FrontoParietal Gamma Synchrony in Patients with Disorders of Consciousness. Brain Topography. 28, 570579.

Cascella, M. (2016). Mechanisms underlying brain monitoring during anesthesia: limitations, possible improvements, and perspectives. Journal of Anesthesiology, 69, 113-120.

Fiset, P.T, Paus, T., Daloze, G., Plourde, G., Meuret, P., Bonhomme, V., Hajj-Ali, N., \& Backman, S.D. (1999). Brain mechanisms of propofol-induced loss of consciousness in humans: A positron emission tomographic study. Journal of Neuroscience, 19, 5506-5513.

Greenblatt, R.E., Pflieger, M.E., \& Ossadtchi, A.E. (2012). Connectivity measures applied to human brain electrophysiological data. Journal of Neuroscience Methods, 207, 1-16.

Guldenmund, P., Vanhaudenhuyse, A., Boly, M., Laureys, S., \& Soddu, A. (2012). A default mode of brain function in altered states of consciousness. Archives Italiennes De Biologie, 150, 107-121.

Gotman, J., Skuce, D.R., Thompson, C.J., Gloor, P., Ives, J.R., \& Ra,y W.F. (1973). Clinical applications of spectral analysis and extraction of features from electroencephalograms with slow waves in adult patients. Electroencephalogry and Clinical Neurophysiology, 35, 225-235.

John, E.R., Prichep, L.S., Kox, W., Valde's-Sosa, P., Bosch-Bayard, J., Aubert, E., Tom, M., diMichele, F., \& Guginoi, L.D. (2001). Invariant Reversible QEEG Effects of Anesthetics. Consciousness and Cognition, 10, 165-183.

Laureys, S., Faymonville, M.E., Janssens, N., Degueldre, C., Peigneux, P., Del Fiore, G., Damas, P., Lambermont, B., Luxen, A., Lamy, M., Moonen, G., \& Maquet, P. (2000). CS4-3.3. Brain function 
during vegetative state and after recovery: A lesional approach to the study of human consciousness. Consciousness and Cognition, 9 (2, pt. 2), S60.

Lehembre, R., Gosseries, O., Lugo, Z., Jedidi, Z., Chatelle, C., Sadzot, B., Laureys, S., \& Noirhomme, G. (2012). Electrophysiological investigations of brain function in coma, vegetative and minimally conscious patients. Archives Italiennes de Biologie, 150, 122-139.

Lodder, S.S., \& van Putten, M.J. (2013). Quantification of the adult EEG background pattern.Clinical Neurophysiology, 124, 228-237.

Sakkalis, V. (2011). Review of advanced techniques for the estimation of brain connectivity measured with EEG/MEG. Computers in Biology and Medicine, 4, 1110-1117.

Thatcher, R.W. (2012). Coherence, Phase Differences, Phase Shift, and Phase Lock in EEG/ERP Analyses. Developmental Neuropsychology, 37, 476-496.

Vatta1, F., Bruno, S., Minine, P., \& Inchingolo, P. (2005). EEG Simulation Accuracy: Reference Choice and Head Models Extension. International Journal of Bioelectromagnetism, 7, 1.

Veselis, R.A, Reinse, R.A., Beattie, B.J., Mawlawi, O.R., Feschenko, V.A., Di Rest, G.R., Larson, S.M., \& Blasberg, R.G., (1997). Midazolam changes cerebral blood flow in discrete brain regions. Anesthesiology, 87, 1106-1117.

Vogt, B.A., \& Laureys, S., (2005). Posterior cingulate, precuneal and retrosplenial cortices: cytology and components of the neural network correlates of consciousness. Progress in Brain Research, 150, 205-217. 